\title{
Estimación del sobrediagnóstico de cáncer mamario en el contexto de un programa de rastreo poblacional con mamografia en Dinamarca
}

Estimation of breast cancer overdiagnosis in the context of a population screening program with mammography in Denmark

\section{Objetivos}

Determinar la asociación entre el tamizaje de cancer de mama y el tamaño de los tumores detectados, y estimar la magnitud de sobrediagnóstico (detección de tumores que no serán clínicamente relevantes) que podría conllevar esta estrategia.

\section{Diseño, lugar y pacientes}

Estudio de cohorte realizado en Dinamarca entre 1980 y 2010 a través de datos anonimizados de todas las mujeres de 35 a 84 años, que para 2010 eran 1.420 .701 .

\section{Descripción de la prueba en estudio y la de referencia} La prueba realizada fue la mamografía, que fue ofrecida bianualmente (con dos incidencias al inicio y una incidencia en el seguimiento) como tamizaje poblacional. Fueron usados datos del grupo danés de cáncer de mama y del registro danés de cáncer para determinar el tamaño del tumor. Los tumores fueron verificados histopatologicamente y fueron considerados no avanzados si eran de $20 \mathrm{~mm}$ o menores y avanzados si eran mayores de $20 \mathrm{~mm}$.

\section{Medición de resultados principales}

La incidencia de los tumores no avanzados en presencia de un tamizaje eficaz, debería reducir la incidencia de los tumores mayores a $20 \mathrm{~mm}$, que son considerados avanzados porque equivalen al nivel T2 en el sistema de clasificación TMN. Por eso, se midió la tendencia en la incidencia de cánceres de mama avanzados y no avanzados en mujeres tamizadas y no tamizadas, a través de dos enfoques para estimar la magnitud del sobrediagnóstico: 1) incidencia total de tumores en mujeres de 50 a 84 años; 2) incidencia de tumores no avanzados en mujeres de 35 a 49 años, 50 a 69 años y 70 a 84 años. Se utilizó la regresión de Poisson para analizar la tendencia de dicha incidencia en diferentes edades, estratificándola por área tamizada y no tamizada. Se comparó la tasa de incidencia para tumores avanzados y no avanzados en áreas tamizadas y no tamizadas, y se calculó el cambio porcentual anual antes y después del tamizaje. En el análisis de mujeres de 50 a 69 años de edad en las áreas no tamizadas, se monitoreó la información para tumores no avanzados en 2007, cuando comenzó el tamizaje a nivel nacional. La tasa de incidencia, las razones de tasas de incidencias y las diferencias de tasas de incidencia se usaron para comparar las tasas antes y después del tamizaje en cada grupo de edad (35 a 49 años, 50 a 69 años y 70 a 84 años) en áreas tamizadas y no tamizadas.

\section{Resultados principales}

No se pudo asociar el tamizaje de cáncer de mama con una menor incidencia de tumores avanzados. La primera aproximación de análisis encontró que 271 tumores invasivos de cáncer de mama y 179 carcinomas ductales in situ (CDIS) fueron sobre diagnosticados en 2010 , con una tasa de sobrediagnóstico de $24,4 \%$ incluyendo CDIS y de $14,7 \%$, excluyéndolo. La segunda aproximación, limitada al análisis de tumores no invasores, documentó que 711 tumores invasores y 180 casos de CDIS fueron sobrediagnosticados en el mismo año, con una tasa de sobrediagnóstico de $48,3 \%$ incluyendo CDIS y de $38,6 \%$, excluyéndolo.

\section{Conclusiones}

El tamizaje de cáncer de mama no estuvo asociado con una reducción en la incidencia de cáncer avanzado en Dinamarca. Es probable que uno de cada tres carcinomas ductales in situ y tumores invasivos diagnosticados en mujeres a quienes se les ofreció tamizaje representen sobrediagnostico, con un incremento de incidencia por sobrediagnóstio de $48,3 \%$.

Fuente de financiamiento: Los autores no reportaron conflictos de interés y trabajan para el Centro Cochrane Nórdico en Dinamarca, la Universidad de Oslo y el Instituto Noruego de Salud Pública.

\section{Comentario}

Este estudio documentó que en Dinamarca la implementación de tamizaje poblacional de cáncer de mama por un tiempo prolongado no redujo la incidencia de tumores avanzados y, por el contrario, incrementó la incidencia de tumores no avanzados y CDIS, no conduciendo a cambios en la mortalidad por cáncer de mama o al uso de tratamientos invasivos para su manejo. El aumento relativo de sobrediagnostico fue estimado en $24,4 \%$, más alto que el de estudios europeos previos, que habían calculado tasas menores, pero con seguimientos menos prolongados ${ }^{1}$. Como ya describió previamente el grupo Cochrane, el tamizaje poblacional con mamografía detecta muchos tumores pequeños que no serán clínicamente relevantes (sobrediagnóstico). Estas lesiones sobrediagnosticadas serán innecesariamente tratadas con cirugía, quimioterapia y radiación, lo que podría exponer a las mujeres a los riesgos de una terapia innecesaria, sin que experimenten beneficios ${ }^{2}$

\section{Conclusiones de los comentadores}

Es importante que los decisores de políticas públicas, los profesionales sanitarios y los usuarios de los sistemas de salud estén al tanto de los riesgos y los beneficios de cualquier medida poblacional, lo que conducirá a que tomen decisiones informadas y a que disminuyan los posibles riesgos para la población.

María Sofía Cuba-Fuentes [Universidad Peruana Cayetano Heredia. maria.cuba@ upch.pe]

Carlos Albrecht Lezama [Universidad Nacional Mayor de San Marcos. albrechtcarlos@gmail.com]

Cuba Fuentes $\mathrm{M}$ y Albrecht $\mathrm{C}$. Estimación del sobrediagnóstico de cáncer mamario en el contexto de un programa de rastreo poblacional con mamografía en Dinamarca. Evid Act Pract Ambul 2017;20(2):53. Comentado de: Jørgensen, K y col. Breast Cancer Screening in Denmark A Cohort Study of Tumor Size and Overdiagnosis. Ann Intern Med. 2017;166:313-323. doi:10.7326/M16-0270. 